\title{
Application of oleanolic acid and its analogs in combating pathogenic bacteria in vitro/vivo by a two-pronged strategy of $\beta$-lactamases and hemolysins
}

\author{
Yonglin Zhou ${ }^{1,2 \#, ~ Y a n ~ G u o ~}{ }^{1,2 \#}$, Xiaodi Sun ${ }^{1}$, Rui Ding ${ }^{1}$, Yanling Wang ${ }^{1,4}$, Xiaodi \\ $\mathrm{Niu}^{3}$, Jianfeng Wang ${ }^{1,2^{*}}$, Xuming Deng ${ }^{1,2^{*}}$
}

${ }^{1}$ Key Laboratory of Zoonosis Research, Ministry of Education, Institute of Zoonosis, College of Veterinary Medicine, Jilin University, Changchun, China

${ }^{2}$ Department of Respiratory Medicine, the First Hospital of Jilin University, Jilin, Changchun, 130021, China,

${ }^{3}$ Department of Food Quality and Safety, Jilin University, Changchun, China ${ }^{4}$ Qingdao Vland biological Limited co., LTD, Qingdao, Shandong, China.

\#Y. Zhou and Y. Guo contributed equally to this work.

*Corresponding Authors: Xuming Deng, College of Veterinary Medicine, Jilin University, Changchun, China, tel.: +86 431-87836161, fax: +86 431-87836161, e-mail: dengxm@jlu.edu.cn; Jianfeng Wang, College of Veterinary Medicine, Jilin University, Changchun, China, tel.: +86 431-87836161, fax: +86 431-87836161, e-mail: wjf927@jilu.edu.cn 
A

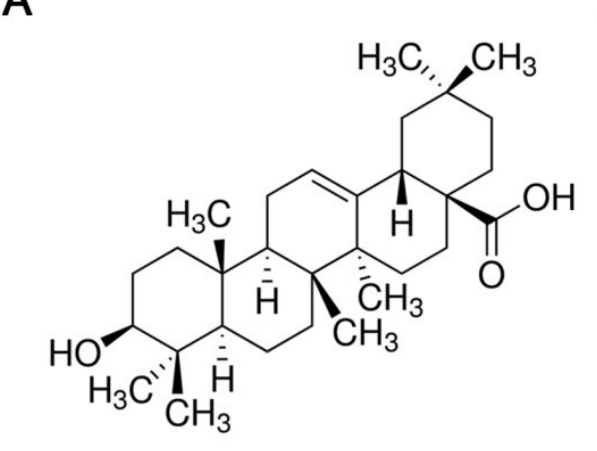

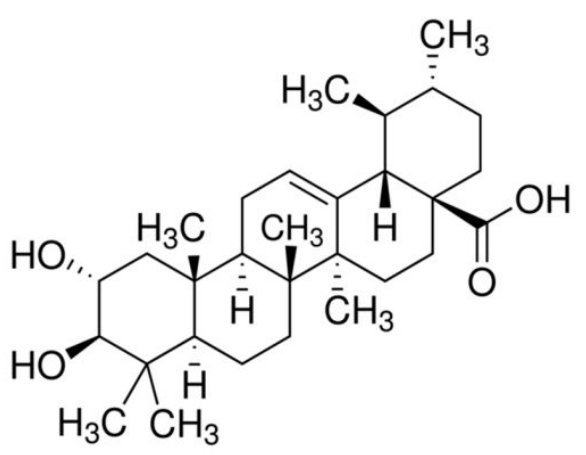

C

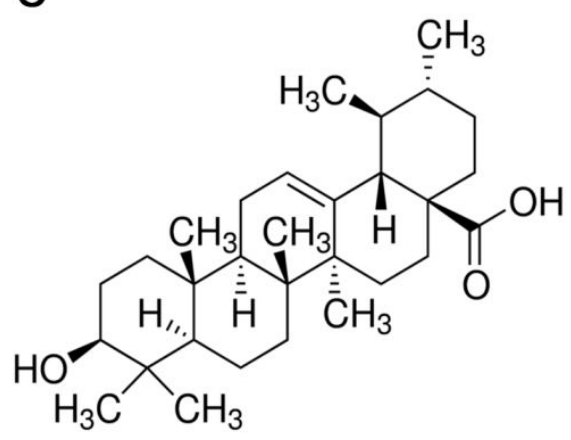

Corosolic Acid

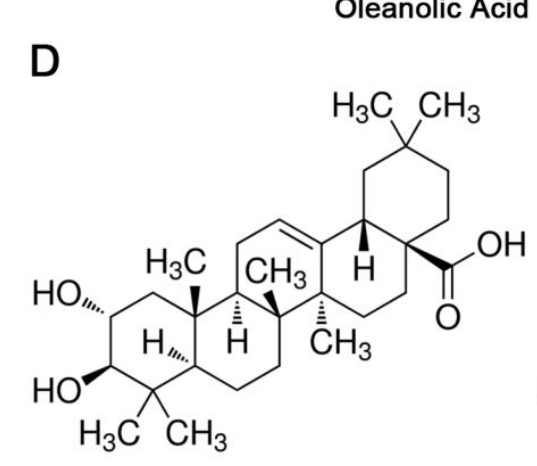

Maslinic Acid

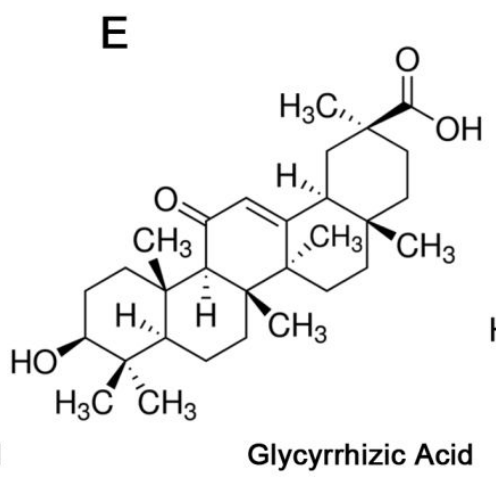

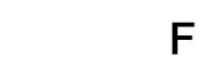

Ursolic Acid

\section{G}

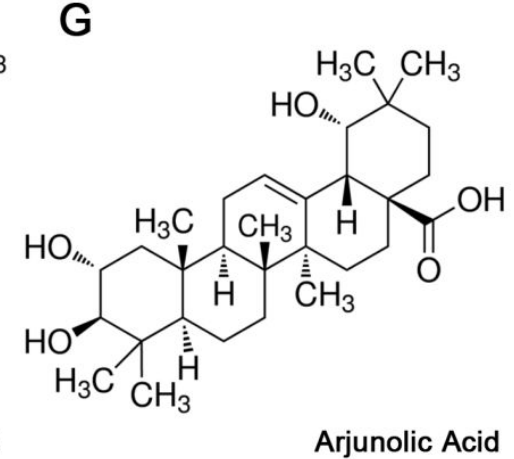

Figure S1. The structure of oleanolic acid and its analogs 
Table S1. MIC values of the different antibiotics and OA combination therapy on Gram-positive bacteria.

\begin{tabular}{|c|c|c|c|c|c|c|c|}
\hline \multirow[t]{9}{*}{ Species } & \multirow{9}{*}{$\begin{array}{l}\beta \text {-lactamase } \\
\text { confirmation }\end{array}$} & \multicolumn{2}{|c|}{$\begin{array}{r}\text { MIC of OA } \\
(\mu \mathrm{g} / \mathrm{mL})\end{array}$} & \multicolumn{3}{|c|}{$\begin{array}{c}\text { MIC of antibiotics } \\
(\mu \mathrm{g} / \mathrm{mL})\end{array}$} & \multirow[t]{2}{*}{ FIC Index } \\
\hline & & Alone & Combination & Antibiotics & Alone & Combination & \\
\hline & & & & Penicillin G & $128(64-128)$ & $8(4-8)$ & $0.19 \pm 0.00$ \\
\hline & & & & Meropenem & $1(1)$ & $0.25(0.125-0.25)$ & $0.33 \pm 0.07$ \\
\hline & & & & Ampicillin Sodium & $64(64-128)$ & $4(4-8)$ & $0.19 \pm 0.00$ \\
\hline & & & & Ceftriaxone Sodium & $64(32-64)$ & $4(4)$ & $0.21 \pm 0.04$ \\
\hline & & & & Ceftiofur Sodium & $16(8-16)$ & $2(2)$ & $0.29 \pm 0.07$ \\
\hline & & & & Cefradine & $128(64-128)$ & $16(16)$ & $0.29 \pm 0.07$ \\
\hline & & $256(256)$ & 32 & Colistin & $128(128)$ & $128(128)$ & $1.13 \pm 0.00$ \\
\hline \multirow{5}{*}{ S. aureus USA300 } & & & & Erythromycin & $32(32)$ & $16(16)$ & $0.63 \pm 0.00$ \\
\hline & + & & & Tetracycline & $256(256)$ & $256(256)$ & $1.13 \pm 0.00$ \\
\hline & & & & Chloramphenicol & $4(4)$ & $2(2)$ & $0.63 \pm 0.00$ \\
\hline & & & & Streptomycin sulfate & $64(32-64)$ & $64(32-64)$ & $1.13 \pm 0.00$ \\
\hline & & & & Kanamycin sulfate & $256(256)$ & $256(256)$ & $1.13 \pm 0.00$ \\
\hline S. aureus ST1027 & + & $256(256)$ & 32 & Penicillin G & $32(16-64)$ & $4(2-4)$ & $0.23 \pm 0.04$ \\
\hline S. aureus ST1053 & + & $256(256)$ & 32 & Penicillin G & $8(8-16)$ & $2(1-4)$ & $0.29 \pm 0.07$ \\
\hline S. aureus ST1057 & + & $256(256)$ & 32 & Penicillin G & $64(32-64)$ & $4(4-8)$ & $0.25 \pm 0.00$ \\
\hline S. aureus ST1058 & + & $256(256)$ & 32 & Penicillin G & $64(32-64)$ & $4(4-8)$ & $0.23 \pm 0.04$ \\
\hline S. aureus ST1061 & + & $256(256)$ & 32 & Penicillin G & $32(32-64)$ & $4(4-8)$ & $0.25 \pm 0.00$ \\
\hline S. aureus ST2022 & + & $256(256)$ & 32 & Penicillin G & $64(32-64)$ & $2(2-4)$ & $0.19 \pm 0.05$ \\
\hline
\end{tabular}




\begin{tabular}{|c|c|c|c|c|c|c|c|}
\hline S. aureus ST2032 & + & $256(256)$ & 32 & Penicillin G & $16(16-32)$ & $1(0.5-1)$ & $0.17 \pm 0.02$ \\
\hline S. aureus ST2064 & + & $512(256)$ & 32 & Penicillin G & $64(64-128)$ & $4(4)$ & $0.11 \pm 0.02$ \\
\hline S. aureus JL01 & + & $256(256)$ & 32 & Penicillin G & $64(32-64)$ & $8(4-8)$ & $0.25 \pm 0.00$ \\
\hline S. aureus JL02 & + & $256(256)$ & 32 & Penicillin G & $32(32)$ & $4(4-8)$ & $0.29 \pm 0.07$ \\
\hline S. aureus ATCC29213 & + & $128(128)$ & 32 & Penicillin G & $4(4)$ & $0.125(0.125)$ & $0.28 \pm 0.00$ \\
\hline S. aureus USA400 & + & $256(256)$ & 32 & Penicillin G & $8(4-8)$ & $1(0.5-1)$ & $0.25 \pm 0.00$ \\
\hline S. aureus 252 & + & $256(128-256)$ & 32 & Penicillin G & $16(16-32)$ & $4(2-4)$ & $0.33 \pm 0.07$ \\
\hline S. aureus 8325-4 & + & $64(64-128)$ & 8 & Penicillin G & $1(0.5-2)$ & $0.125(0.125)$ & $0.21 \pm 0.07$ \\
\hline S. aureus ATCC25904 & + & $256(256)$ & 64 & Penicillin G & $64(64)$ & $1(1)$ & $0.27 \pm 0.00$ \\
\hline S. aureus ATCC25923 & - & $32(16-32)$ & 4 & Penicillin G & $0.01(0.01)$ & $0.01(0.01)$ & $1.17 \pm 0.07$ \\
\hline
\end{tabular}


Table S2. MIC values of the different antibiotics and OA combination therapy on Gram-negative bacteria.

\begin{tabular}{|c|c|c|c|c|c|c|c|}
\hline \multirow[t]{2}{*}{ Species } & \multirow{2}{*}{$\begin{array}{l}\beta \text {-lactamase } \\
\text { confirmation }\end{array}$} & \multicolumn{2}{|c|}{$\begin{array}{r}\text { MIC of OA } \\
(\mu \mathrm{g} / \mathrm{mL})\end{array}$} & \multicolumn{3}{|c|}{$\begin{array}{c}\text { MIC of antibiotics } \\
(\mu \mathrm{g} / \mathrm{mL})\end{array}$} & \multirow[t]{2}{*}{ FIC Index } \\
\hline & & Alone & Combination & Antibiotics & Alone & Combination & \\
\hline \multirow{8}{*}{ E.coli ZJ487 (NDM-1/MCR-1) } & \multirow{8}{*}{+} & \multirow{8}{*}{$512(\geq 512)$} & \multirow{8}{*}{32} & Meropenem & $32(16-32)$ & $2(2)$ & $0.17 \pm 0.04$ \\
\hline & & & & Gentamycin sulfate & $512(\geq 512)$ & $512(\geq 512)$ & $1.06 \pm 0.00$ \\
\hline & & & & Erythrocin & $128(64-128)$ & $128(64-128)$ & $1.06 \pm 0.00$ \\
\hline & & & & Tetracycline & $128(128)$ & $128(128)$ & $1.06 \pm 0.00$ \\
\hline & & & & Chloramphenicol & $2(2)$ & $2(2)$ & $1.06 \pm 0.00$ \\
\hline & & & & Streptomycin sulfate & $64(64-128)$ & $128(64-128)$ & $1.06 \pm 0.00$ \\
\hline & & & & Kanamycin sulfate & $128(128)$ & $128(128)$ & $1.06 \pm 0.00$ \\
\hline & & & & Colistin & $8(8)$ & $8(8)$ & $1.06 \pm 0.00$ \\
\hline E. coli ZC-YN3 (NDM-1) & + & $512(\geq 512)$ & 32 & Meropenem & $32(16-32)$ & $4(2-4)$ & $0.19 \pm 0.00$ \\
\hline E. coli ZC-YN5 (NDM-5) & + & $512(\geq 512)$ & 64 & Meropenem & $64(32-64)$ & $4(4-8)$ & $0.23 \pm 0.04$ \\
\hline E. coli ZC-YN7 (NDM-9) & + & $512(\geq 512)$ & 64 & Meropenem & $32(32)$ & $4(4)$ & $0.25 \pm 0.00$ \\
\hline K. pneumoniae QD-KP2 (NDM-1) & + & $512(\geq 512)$ & 32 & Meropenem & $64(64-128)$ & $4(4-8)$ & $0.13 \pm 0.00$ \\
\hline E.coli D3 (NDM-1/OXA-1) & + & $512(256-512)$ & 32 & Meropenem & $32(32)$ & $8(8)$ & $0.33 \pm 0.04$ \\
\hline E.coli E2 (NDM-9/OXA-10) & + & $512(\geq 512)$ & 32 & Meropenem & $16(16)$ & $4(2-4)$ & $0.27 \pm 0.07$ \\
\hline E.coli E4 (NDM-5/OXA-1) & + & $256(256)$ & 32 & Meropenem & $32(32-64)$ & $8(8)$ & $0.33 \pm 0.07$ \\
\hline E. coli BL21 (pET28a-SP-NDM-1) & + & $512(\geq 512)$ & 32 & Meropenem & $64(32-64)$ & $4(4-8)$ & $0.17 \pm 0.04$ \\
\hline \multirow{2}{*}{ E. coli BL21 (pET28a-KPC-2) } & \multirow[t]{2}{*}{+} & \multirow[t]{2}{*}{$512(\geq 512)$} & \multirow[t]{2}{*}{32} & Meropenem & $2(1-2)$ & $0.25(0.25-0.5)$ & $0.27 \pm 0.07$ \\
\hline & & & & Imipenem & $16(8-16)$ & $2(2)$ & $0.23 \pm 0.07$ \\
\hline
\end{tabular}




\begin{tabular}{|c|c|c|c|c|c|c|c|}
\hline E. coli BL21 (pET28a-VIM-1) & + & $512(\geq 512)$ & 32 & Cefalotin sodium & $64(64)$ & $16(16-32)$ & $0.40 \pm 0.14$ \\
\hline E. coli BL21 (pET28a-TEM-1) & + & $512(\geq 512)$ & 32 & Cefalotin sodium & $8(8-16)$ & $4(4-8)$ & $0.65 \pm 0.39$ \\
\hline E. coli BL21 (pET28a-OXA-1) & + & $512(\geq 512)$ & 32 & Imipenem & $0.06(0.06)$ & $0.06(0.06)$ & $1.06 \pm 0.00$ \\
\hline E. coli BL21 (pET28a) & - & $512(\geq 512)$ & 32 & Meropenem & $0.008(0.008)$ & $0.008(0.008)$ & $1.06 \pm 0.00$ \\
\hline
\end{tabular}

All the data of MIC values were the median (range for the data), $n=3$. The column of strains information showed originally carried or established $\beta$-lactamase in parentheses. 
Table S3. The primers of all the $\beta$-lactamases for normal pcr used in this study.

\begin{tabular}{|c|c|c|}
\hline Gene names & Primer names & Oligonucleotide primer sequence ( $\left.5^{\prime}-3^{\prime}\right)$ \\
\hline \multirow{2}{*}{$n d m-1$} & $n d m-1-F$ & ctgggatccatggaattgeccaatattatg \\
\hline & $n d m-1-R$ & ctgetcgagtcagcgcagcttgtcgg \\
\hline \multirow{2}{*}{$s p-n d m-1$} & $s p-n d m-1-F$ & cgggatcettgaattcgccccatat \\
\hline & $s p-n d m-1-R$ & ccetcgagtcagcgcagcttgtcggccat \\
\hline \multirow{2}{*}{$k p c-2$} & $k p c-2-F$ & ctgggatccatggcggaaccattcgcta \\
\hline & $k p c-2-R$ & ctggtcgacttactgeccgttgacg \\
\hline \multirow{2}{*}{ vim-1 } & vim-1-F & ctgggatccatgttaaaagttattagtagtttattg \\
\hline & vim-1-R & ctgetcgagctactcggegactgag \\
\hline \multirow{2}{*}{$\beta$-lactamase-N1 } & $\beta$-lactamase-N1-F & cgcggatccatgagtttaataaagaaaaagaataaag \\
\hline & $\beta$-lactamase-N1-R & ccgetcgagttaaatttcagaaattactggaataat \\
\hline \multirow{2}{*}{ oxa-1 } & oxa-1-F & cgcggatcettgttagccgttaaaattaagccc \\
\hline & $o x a-1-R$ & ccgctcgagttataaatttagtgtgtttagaatggt \\
\hline \multirow{2}{*}{ tem-1 } & tem-1-F & ctgggatcccacccagaaacgctgg \\
\hline & tem-1-R & ccgctcgagttaccaatgcttaatcagtgagg \\
\hline
\end{tabular}

